\title{
Limited Distal Arch Open Stenting for Type B Aortic Dissection with an Enlarged Ulcer-Like Projection, Complicated by Abdominal Aortic Occlusion
}

\author{
Mitsumasa Hata ${ }^{1}$ Kenji Akiyama ${ }^{1} \quad$ Yukihiko Orime $^{1}$ \\ ${ }^{1}$ Department of Cardiovascular Surgery, Nihon University Hospital, \\ Tokyo, Japan \\ Thorac Cardiovasc Surg Rep 2016;5:71-73.

\begin{abstract}
Address for correspondence Mitsumasa Hata, MD, PhD, Department of Cardiovascular Surgery, Nihon University Hospital, 1-6 Kanda, Surugadai, Chiyoda-ku, Tokyo 101-8309, Japan

(e-mail: hata.mitsumasa@nihon-u.ac.jp).
\end{abstract}

\begin{abstract}
A 79-year-old man underwent bifurcated graft replacement for abdominal aortic aneurysm, but the graft was totally occluded owing to an acute type B aortic dissection. We could not have access to the root for performing endovascular stenting for

Keywords

- aortic aneurysm

- cardiovascular surgery

- aortic stent dissecting aneurysm. Furthermore, it was difficult to remove the aneurysm via a left thoracotomy because it was impossible to perfuse the lower body with blood from the femoral artery and also a left axillary arterial cannulation was unusable. Herein, we report a patient who was successfully treated with open surgical aortic stenting for an enlarged ulcer-like projection complicated by a type B aortic dissection.
\end{abstract}

\section{Introduction}

Management of distal arch pathology is problematic. Recently, thoracic endovascular aortic repair (TEVAR) has become an attractive method for treating arch aneurysms. ${ }^{1}$ However, newly developed techniques for open surgical repair of arch aneurysms and aortic dissection have resulted in increased positive outcomes in recent years. ${ }^{2}$ Herein, we report a patient who was successfully treated with open surgical aortic stenting for an enlarged ulcer-like projection (ULP) complicated by a type B aortic dissection.

\section{Case Report}

The patient was a 79-year-old man who underwent bifurcated graft replacement for abdominal aortic aneurysm in 2013 at age 77 years. One year later, the bifurcated graft was totally occluded with large thrombus formed owing to an acute type B aortic dissection. A bilateral axillofemoral bypass was performed to relieve the leg ischemia. Followup computed tomography (CT) scan revealed patent axillofemoral bypass and an enlarged ULP (localized contrast media in the occluded false lumen showing the primary entry site) on the distal aortic arch (-Fig. 1). Coronary CT angiography showed significant stenosis of the left anterior descending artery (LAD). We performed LAD bypass surgery using a saphenous vein graft and an open stent implantation through a median sternotomy during mild hypothermic circulatory arrest. Circulatory arrest was induced at a rectal temperature of $28^{\circ} \mathrm{C}$ without cerebral perfusion. The hemicircumference of the anterior surface of the arch between the left common carotid and left subclavian arteries was obliquely incised. A J-Graft Open Stent (Japan Lifeline Co., Ltd., Tokyo, Japan) was inserted into the descending aorta to cover the ULP. The proximal side of the graft was trimmed and continuously sutured around the orifice of the left subclavian artery. The aortic incision and anterior side of the graft were closed using single continuous suture. The durations of circulatory arrest, cross-clamp, cardiopulmonary bypass, and overall surgery were 16, 39, 103, and 173 minutes, respectively. The patient was discharged without any complications on postoperative day 10 . Postoperative CT scans showed a patent coronary bypass and no end leakage from the suture line (-Fig. 2). received

October 20, 2015

accepted after revision

November 6, 2015

published online

January 5, 2016
DOI http://dx.doi.org/

10.1055/s-0035-1570377. ISSN 2194-7635. (c) 2016 Georg Thieme Verlag KG
Stuttgart · New York

License terms

(c) $(1) \$$ 

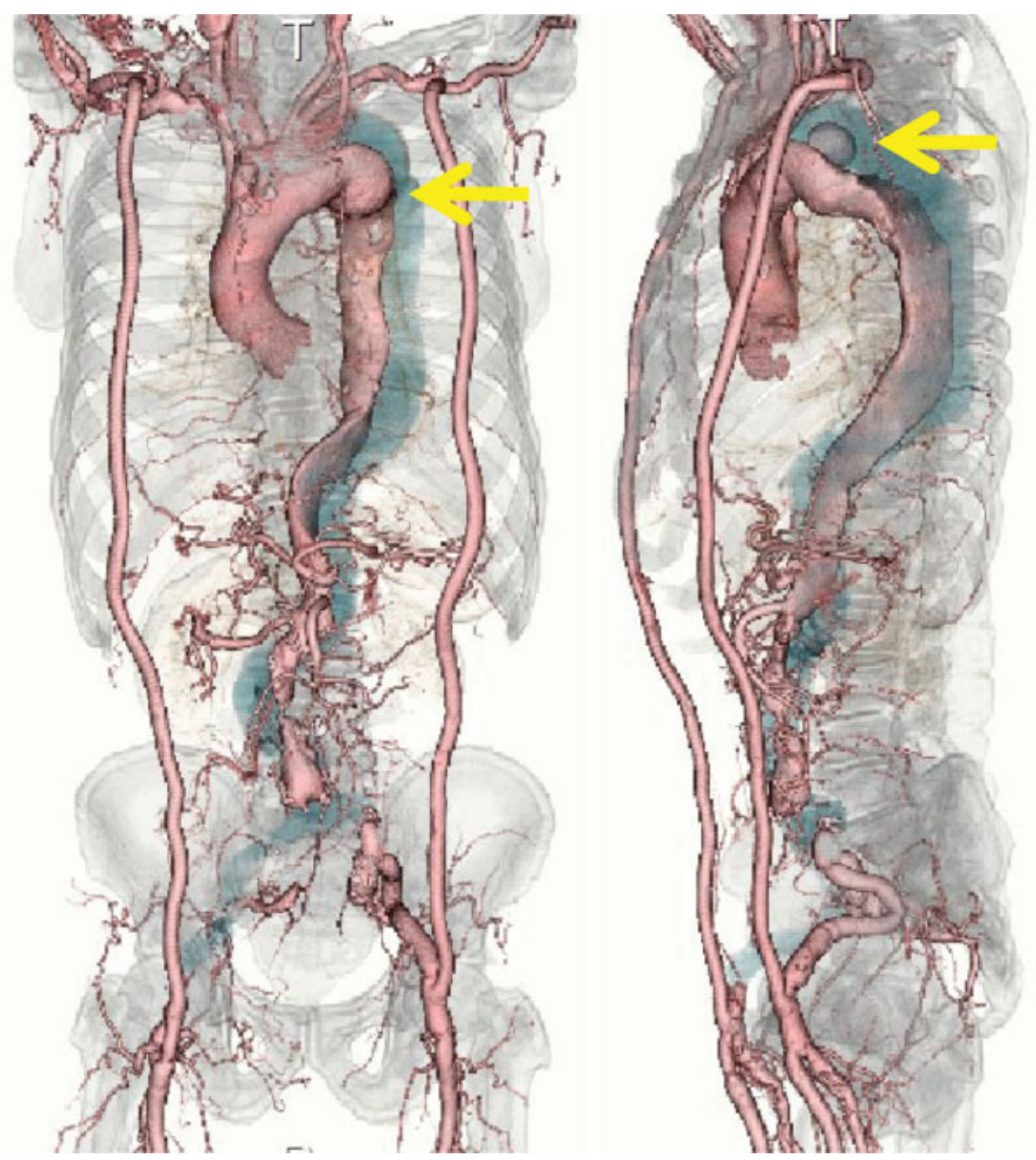

Fig. 1 Preoperative CT finding. Preoperative CT scans revealed patent axillofemoral bypass and an enlarged ulcer-like projection (arrow) located on the distal aortic arch.

\section{Discussion}

In the present case, the primary entry closure and redo Y-graft replacement should be performed at the time of dissection onset. However, the patient was so sick due to blood acidosis associated with lower body malperfusion at the time of dissection onset. Therefore, we considered that the patient might not be able to tolerate aggressive surgical stress of redo Y-graft replacement under general anesthesia. Therefore, we selected bilateral axillofemoral bypass under local anesthesia to relieve the lower body ischemia at the time of type $B$ dissection onset. And then we had to fix the enlarging ULP in the next step. Recently, TEVAR has been recommended as the first line of treatment for type B aortic dissections, even when no complications exist, because of its improved early outcomes compared with open surgery. ${ }^{3}$ In the present case, it would have been safer and easier to fix the ULP by TEVAR if we could have accessed the root for performing TEVAR. On the other hand, we recently demonstrated the benefits of open repair using left thoracotomy for treating type B dissecting aneurysms. ${ }^{4}$ However, it was difficult to remove the long segment of the dissecting aneurysm via a left thoracotomy approach because it was impossible to perfuse the lower body with blood from the femoral artery owing to occlusion of the abdominal aorta. A left axillary arterial cannulation was also unusable because we had to clamp the left subclavian artery and the aortic arch between the left common carotid and subclavian arteries. Furthermore, total arch replacement using antegrade cerebral perfusion would have risked axillofemoral bypass steal syndrome, reducing blood flow to the brain. In this case, our unique and less invasive method using open stenting with mild hypothermic circulatory arrest and no cerebral perfusion was the most appropriate procedure. ${ }^{5}$ Cerebral perfusion was not needed, because circulatory arrest was completed within 20 minutes. Furthermore, this approach avoids the risks of phrenic or recurrent nerve injury, as well as pulmonary complications. Therefore, we consider this procedure suitable for minimizing cardiopulmonary bypass time when concomitant open heart surgery is required. 


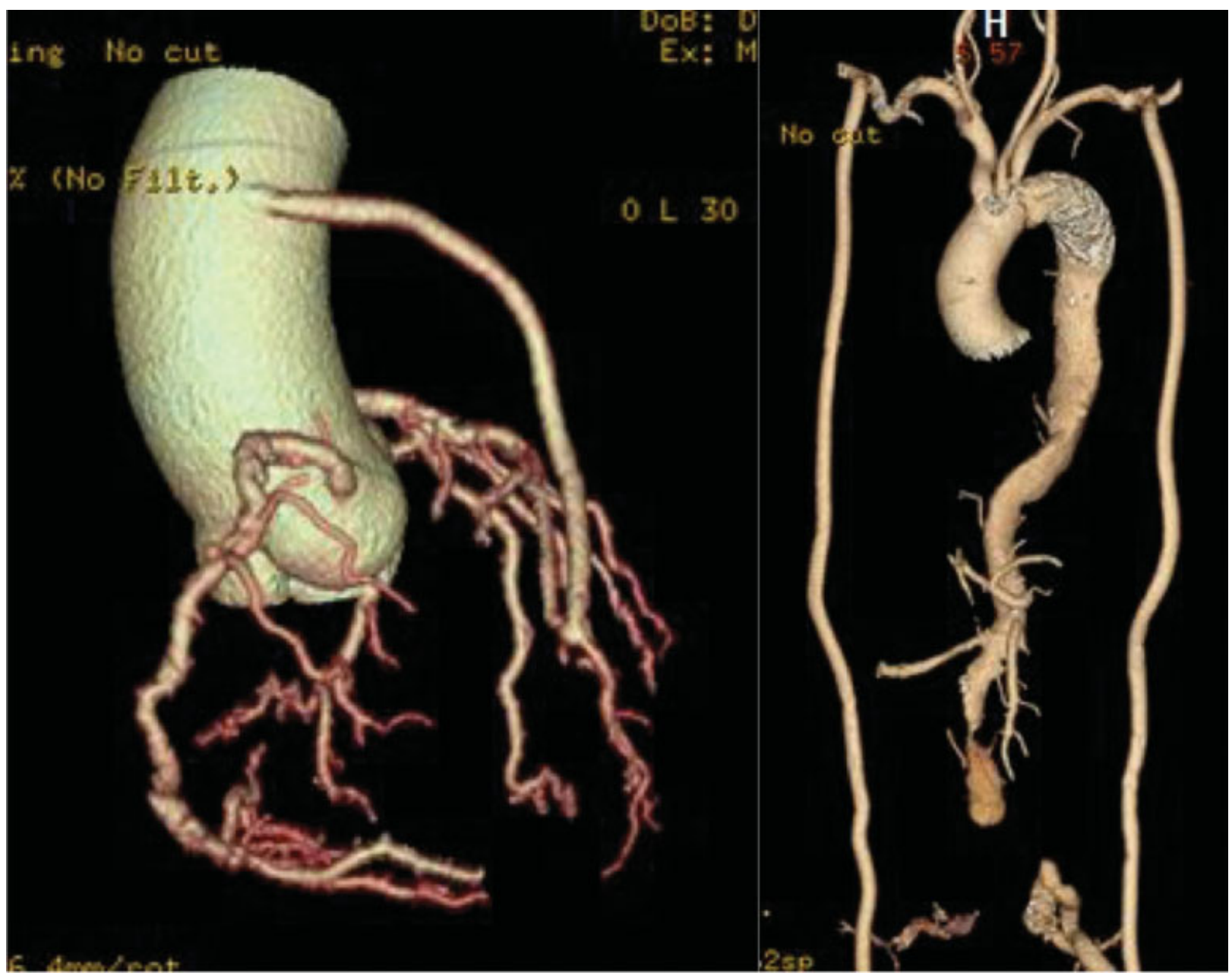

Fig. 2 Postoperative CT findings. Postoperative CT scans showed patent coronary bypass (left) and no end leakage from the suture line (right).

\section{Conclusion}

Herein, we report a rare case of a complicated distal arch pathology successfully treated by limited surgical open stenting.

Conflict of Interest

None.

\section{References}

1 Ishida M, Kato N, Hirano T, Cheng SH, Shimono T, Takeda K. Endovascular stent-graft treatment for thoracic aortic aneurysms: short- to midterm results. J Vasc Interv Radiol 2004;15(4):361-367
2 Estrera AL, Sandhu H, Afifi RO, et al. Open repair of chronic complicated type $\mathrm{B}$ aortic dissection using the open distal technique. Ann Cardiothorac Surg 2014;3(4): 375-384

3 Brunkwall J, Lammer J, Verhoeven E, Taylor P. ADSORB: a study on the efficacy of endovascular grafting in uncomplicated acute dissection of the descending aorta. Eur J Vasc Endovasc Surg 2012;44(1):31-36

4 Hata M, Orime Y, Akiyama K, et al. Outcomes of open surgical repair for type $B$ dissecting aortic aneurysm with alternative methods in the endovascular stent era. Semin Thorac Cardiovasc Surg 2015;27(2):106-112

5 Hata M, Akiyama K, Orime Y, Wakui S, Nakamura T, Shiono M. Less invasive quick open stenting using A J Graft Open Stent for distal arch aneurysms. Thorac Cardiovasc Surg 2016;64(4): 330-332 